\title{
Correlation Analysis for Selection of Microtitanium Plates with Different Specifications for Use in a Cervical Vertebral Dome Expansion Laminoplasty
}

\author{
Run-tian Zhou, MB, Xiao-feng Zhao, MM, Yi-bo Zhao, MM, Xiang-dong Lu, MM, Zhi-feng Fan, MM, De-tai Qi, MM, \\ Xiao-nan Wang, MB, Yuan-zhang Jin, MB, Bin Zhao, MD (D) \\ The Second Affiliated Hospital of Shanxi Medical University, Taiyuan, China
}

\begin{abstract}
Objective: To analyze correlations between the selection of microtitanium plates with different specifications for use in a cervical vertebral dome expansion laminoplasty.

Methods: Sixteen patients that underwent the cervical vertebral dome expansion laminoplasty with a cervical spinal stenosis angioplasty procedure for treatment of their cervical spinal cords were recruited at our hospital. From February 2017 to September 2018, medical records confirmed that all patients underwent cervical CT and MRI tests pre- and postsurgery. The anteroposterior diameter of the spinal canal, changes in the cross-sectional area of the spinal canal, and the pre- and postsurgery distance of the cervical spinal cord after applying microtitanium plates with different lengths were measured by Mimics version 17.0 software (Materialise NV, Leuven, Belgium). A statistical regression and correlation analysis of relevant specification parameters of the microtitanium plate was then studied.
\end{abstract}

Results: As the size of the microtitanium plate increased, we found that the cross-sectional area of cervical spinal canal and distance between the descendants of the lamina and the distance of cervical spinal cord concordantly increased, and these data changes linearly. The regression equation associated with sagittal diameter, crosssectional area, and posterior movement distance of the cervical spinal cord was obtained.

Conclusion: According to the correlation analysis of imaging data changes, the regression equation was obtained to guide the selection of microtitanium plates with appropriate specifications in a cervical vertebral dome expansion laminoplasty.

Key words: Cervical spinal stenosis; Laminar back distance; Medical imaging measurement; Miniature titanium plate; Spinal canal enlargement

\section{Introduction}

With the progression of modern society, cervical spondylosis has appeared to be one of the most common orthopedic diseases. It is caused by a reduction in the anteroposterior diameter and effective volume of the spinal canal $^{1}$. Generally, patients with severe symptoms and signs need surgical treatment ${ }^{2}$. In 1977, Hirabayashi et al. ${ }^{3}$ first proposed an internal fixation of a single open-door miniature titanium plate. After years of development, the internal fixation of a single open-door miniature titanium plate has achieved good clinical results and is now recognized by the majority of orthopedic specialists as one of the most commonly used methods for treating cervical spondylosis-related diseases. With the improvement of posterior cervical surgery, many scholars have improved and optimized the associated surgical methods.

Our team has also improved in this context. According to the principle and purpose of cervical spinal canal osteoplasty in the treatment of cervical spondylopathy, we have finally developed a cervical vertebral dome expansion laminoplasty. This type of surgery involves lavage of the lamina at the junction of the $\mathrm{C}_{3}-\mathrm{C}_{7}$ bilateral lamina and the facet

Address for correspondence Bin Zhao, MD, The Second Affiliated Hospital of Shanxi Medical University, Taiyuan, China. Email: zzbb3737@126.com Received 8 March 2021; accepted 17 May 2021 
OrthopaEdic SURGery

VOLUMe $13 \cdot$ NUMBer $7 \cdot$ OCTOBER, 2021
Cervical Vertebral Dome Expansion Laminoplasty

the distance of the posterior lamina movement is too large, the spinal cord will excessively drift backward, and the nerve roots will be significantly pulled as a result, which might then cause the occurrence of $\mathrm{C}_{5}$ nerve root palsy symptoms. According to studies by Lubelski et al., the cervical spinal canal diameter is enlarged by $1 \mathrm{~mm}$, and the probability of $\mathrm{C}_{5}$ nerve root paralysis is increased by approximately $69 \%$. Therefore, an appropriate lamina posterior distance is crucial if clinical efficacy and recovery from the surgical procedure is to be realized. In addition, the distance of the posterior lamina movement depends on the structure of the vertebral body and the specifications of the custom microtitanium plate. The choice of differently sized microtitanium plates for different cervical segments will influence the achievement of different posterior lamina distances. Thus, a selection of suitable microtitanium plates for different internal cervical segments to enable strong internal fixation represents an important part and focus of study.

In the current study, we measured and analyzed CT and MRI data of 16 patients with the cervical vertebral dome expansion laminoplasty at our medical center. We measured the distance of the posterior lamina, the enlarged area of the spinal canal, and the drift distance of the spinal cord. The intent of the study was therefore to explore the correlation between the different types of microtitanium plates in different cervical segments in terms of the above three parameters. We also sought to establish guidance for the selection of suitable microtitanium plates in the setting of a posterior tracheal angioplasty with the aim of achieving improved surgical results and clinical efficacy.

Through the data analysis and correlation study of the changes in the cervical spine imaging data of these 16 patients underwent the cervical vertebral dome expansion laminoplasty, the purpose of this study is: (i) to clarify the cal symptoms do not significantly improve. Additionally, if
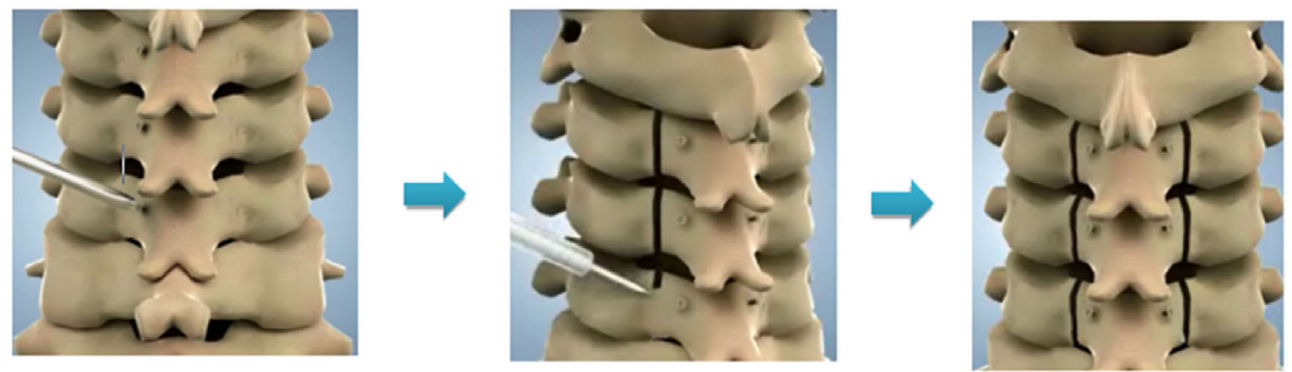

Fig. 1 Cervical vertebral dome expansion laminoplasty procedure diagram. This operation first cuts off both sides of the cervical lamina, and then moves the entire lamina back, and then fixes the cervical spinal cord with a titanium plate.
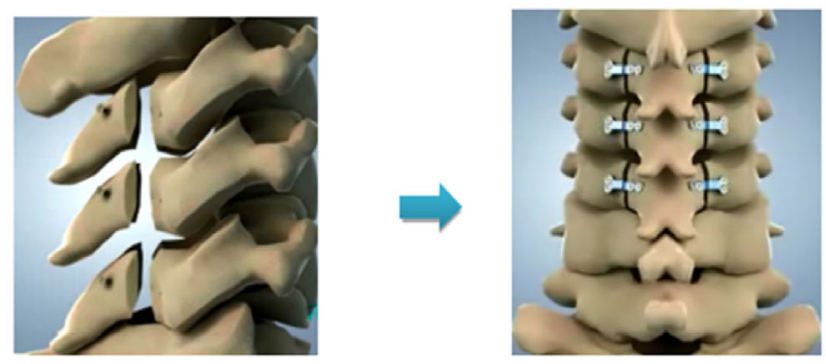
ORTHOPAEDIC SURGERY Volume $13 \cdot$ Number 7 • OCTOBer, 2021
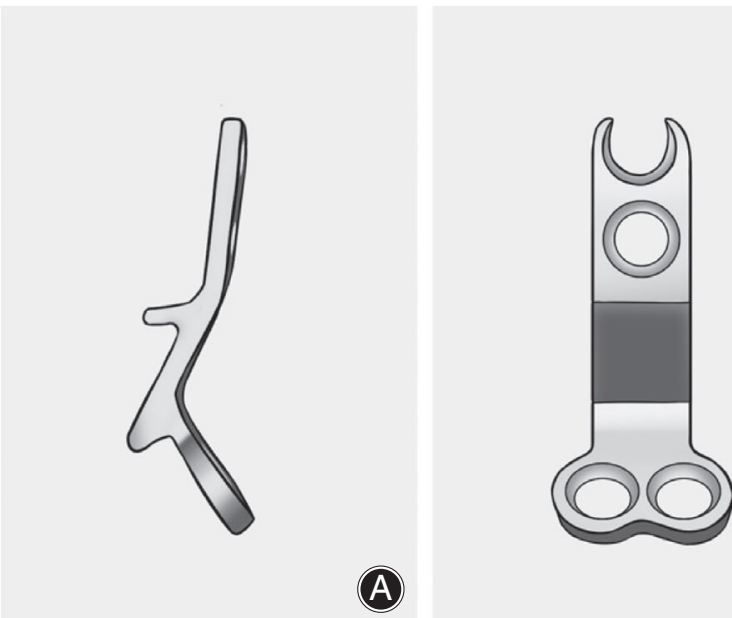

(A)

(B)

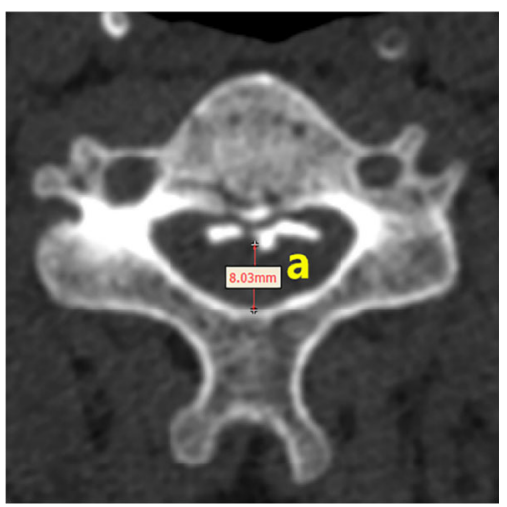

Fig. 3 Preoperative spinal anteroposterior diameter a. To measure the midpoint of the posterior margin of the vertebral body or the pathological mass to the midpoint of the posterior border of the spinal canal, which were recorded as "a".

correlation between different specifications of titanium plates and changes in the imaging data of the cervical spinal canal; (ii) to obtain the regression equation between the use of different specifications of titanium plates and the imaging changes of the cervical spinal canal; and (iii) to guide the selection and application of microtitanium plates specifications in a cervical vertebral dome expansion laminoplasty.

\section{Materials and Methods}

\section{General Information}

In February 2017 through September 2018, 16 patients that underwent the cervical vertebral dome expansion laminoplasty for the treatment of their cervical spinal cord were selected and recruited to the study on the basis of their medical records. The patients included 14 males and 2 females, which were aged $49-76$ years, with a mean age of $57.3 \pm 1.7$ years. All 16 patients underwent cervical CT and

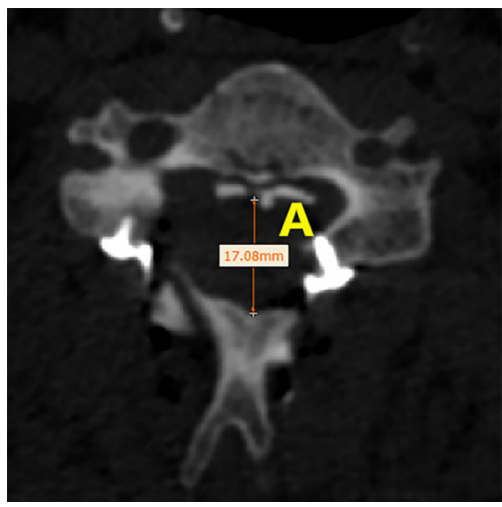

Fig. 4 Postoperative spinal anteroposterior diameter A. To measure the sagittal diameter of the spinal canal after completing a cervical vertebral dome expansion laminoplasty, which was recorded as "A".

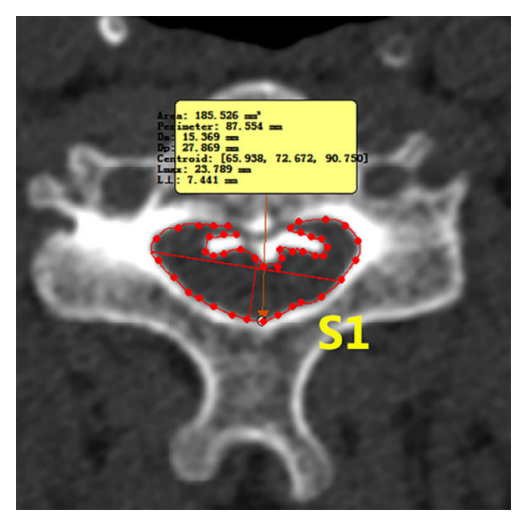

Fig. 5 Preoperative spinal canal cross-sectional area $\mathrm{S}_{1}$. The crosssectional area which the posterior border was the leading edge of the segmental lamina, and the inner edge of the pedicle on both sides served as the boundary between those sides was recorded as $S_{1}$. 
ORTHOPAEDIC SURGERY

Volume $13 \cdot$ Number $7 \cdot$ OCtober, 2021
Cervical Vertebral Dome Expansion Laminoplasty

application, and their dimensions are listed as follows: $27 \times 4.5 \times 1$; 4 holes, $29 \times 4.5 \times 1$; 4 holes, and $31 \times 4.5$ $\times 1 ; 4$ holes (Fig. 2).

the DICOM format by a 0.5 -row thin-layer scan of a 64 -row CT machine.

Different types of custom cervical posterior microtitanium plates were developed and selected for intraoperative

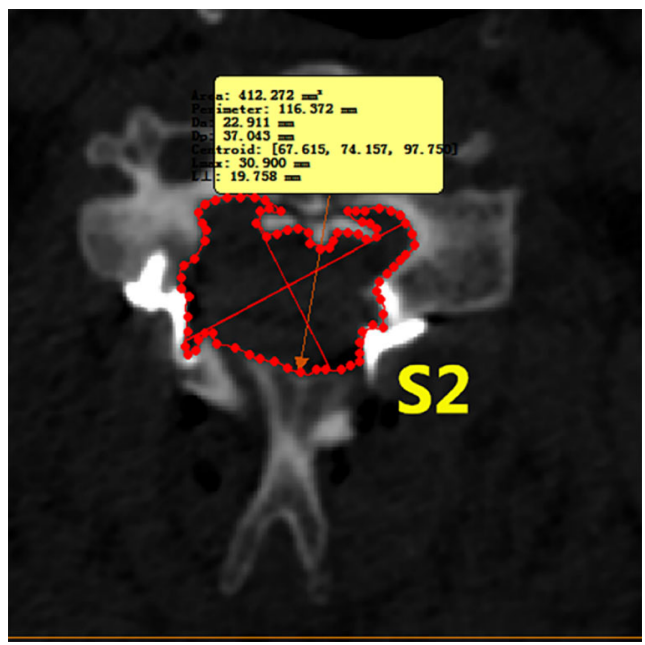

Fig. 6 Postoperative spinal canal cross-sectional area S2. The crosssectional area of the spinal canal after the surgery is recorded as S2.

\section{Inclusion and Exclusion Criteria}

\section{Inclusion Criteria}

The inclusion criteria were as follows: (i) patients met the symptoms and signs of cervical spinal stenosis, confirmed by imaging and neurophysiological examinations; (ii) patients treated with cervical vertebral dome expansion laminoplasty; (iii) patients who completed preoperative and postoperative imaging examinations; (iv) the main evaluation indicators included diameter of the spinal canal, cross-sectional area of the spinal canal; and (v) a correlation research study.

\section{Exclusion Criteria}

The exclusion criteria were as follows: (i) patients with heart, brain, kidney, and other important organ diseases; (ii) patients with cervical spinal stenosis caused by acute trauma, spinal canal occupying lesions, and infection; and (iii) patients unable to cooperate with surgical treatment.
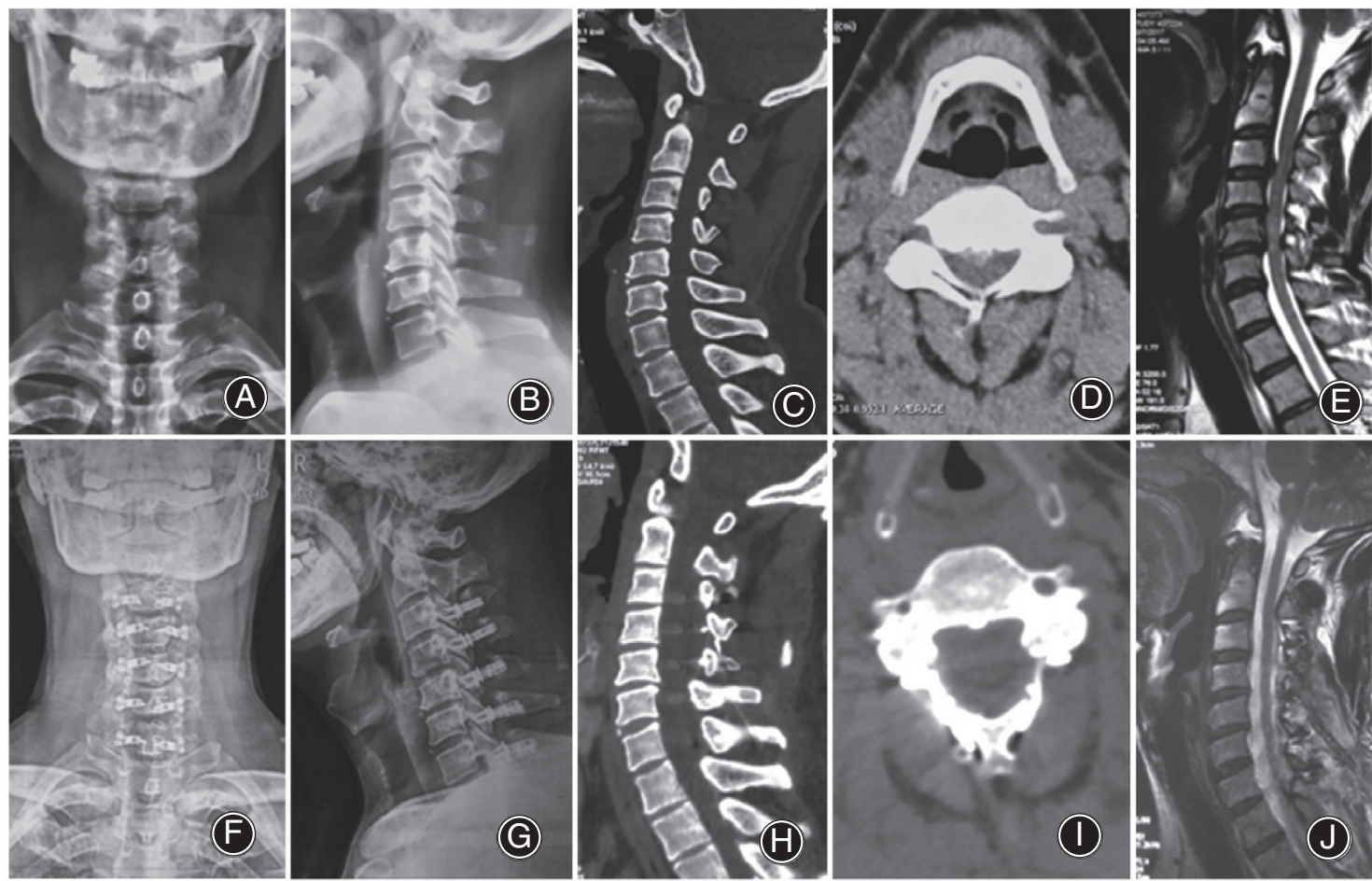

Fig. 7 A 54-year-old male with numbness and weakness in both upper limbs for 2 years was diagnosed with cervical spondylosis. (A and B) showed the condition before the cervical spine surgery, ( $F$ and $G$ ) showed the condition after the cervical spine surgery. (C, D and E) showed the cervical spinal canal stenosis, and the cervical spinal cord was compressed obviously. $(\mathrm{H}, \mathrm{I}$ and $\mathrm{J}$ ) showed that after cervical surgery, the cervical spinal canal was significantly enlarged and the spinal cord was no longer compressed. 


\section{Experimental Methods}

The obtained DICOM data from the pre- and postoperative CT (DICOM formatted data was obtained from a 0.5 -row CT $0.5-\mathrm{mm}$ thin-layer scan) were imported into Mimics version 17.0 software for measuring the desired data, including the measurement of the anteroposterior diameter of the spinal canal and the cross-sectional area of the spinal canal when using a small titanium plate in different cervical segments. The distance of the spinal cord drifting back in different cervical segments was measured using Photoshop CS5 software (Adobe Inc., San Jose, California).

\section{Data of the Cervical Posterior Lamina Retraction Distance}

Mimics version 17.0 software was used to: (i) measure the sagittal diameter of the midpoint of the vertebral pedicle in the midpoint of the $\mathrm{C}_{3-7}$ segment; and (ii) measure the midpoint of the posterior margin of the vertebral body or the pathological mass to the midpoint of the posterior border of the spinal canal, which were recorded as "a." The same method was used to measure the sagittal diameter of the spinal canal after completing a cervical vertebral dome expansion laminoplasty, which was recorded as "A." Thus, the lamina back movement distance was determined as "A-a"(Figs 3 and 4)..

\section{Data of the Change in Cervical Spinal Canal Cross- Sectional Area}

Using Mimics version 17.0 software to measure the crosssectional area (CSA) of the midpoint plane of the cervical vertebral pedicle in each segment of $\mathrm{C}_{3-7}$, we found that when taking measurements, the anterior border of the spinal canal was the posterior edge of the vertebral body or the trailing edge of the pathological mass. In addition, the posterior border was the leading edge of the segmental lamina, and the inner edge of the pedicle on both sides served as the boundary between those sides. The cross-sectional area was recorded as S1, and the same method was used to measure the CSA of the enlarged spinal canal after the new, full backward shift of the lamina in the spinal canal, which was recorded as S2. The increased CSA of the spinal canal was recorded as S2-S1 (Figs 5 and 6).

\section{Data of the Spinal Cord Posterior Distance in the Cervical Spinal Canal}

Measuring the distance of the spinal cord by Photoshop CS5 software, we used the midpoint of the posterior wall of the vertebral body as the starting point. Alternatively, if there was an occupying pathological tissue, the midpoint of the occupying tissue in the spinal canal was used as the starting point of measurement. Using the sagittal midpoint of the cervical spinal cord as the measurement endpoint, we recorded the distance between both of these points. The distance from the midpoint of the spinal cord to the posterior wall of the vertebral body was recorded and defined as " $c$ "; the same method was used to measure the distance between the two points by MRI after completing the cervical vertebral dome expansion laminoplasty, wherein the distance

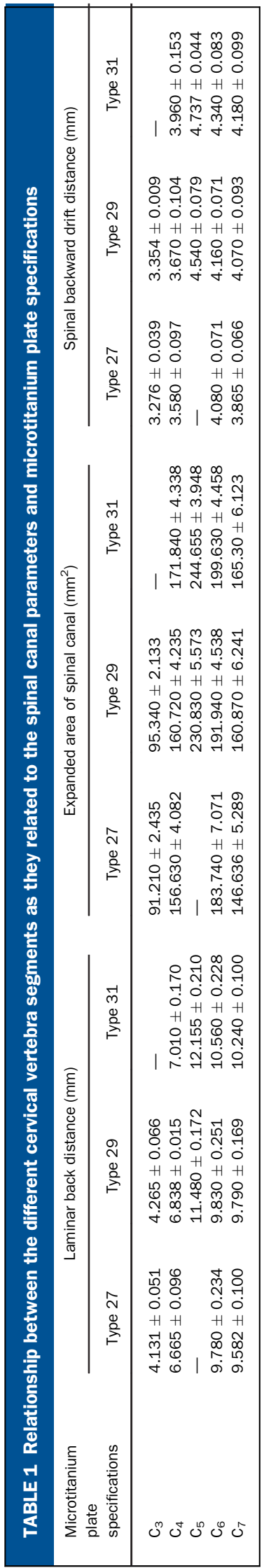


Fig. 8 Number of different titanium plates used in different segments of the cervical vertebra.

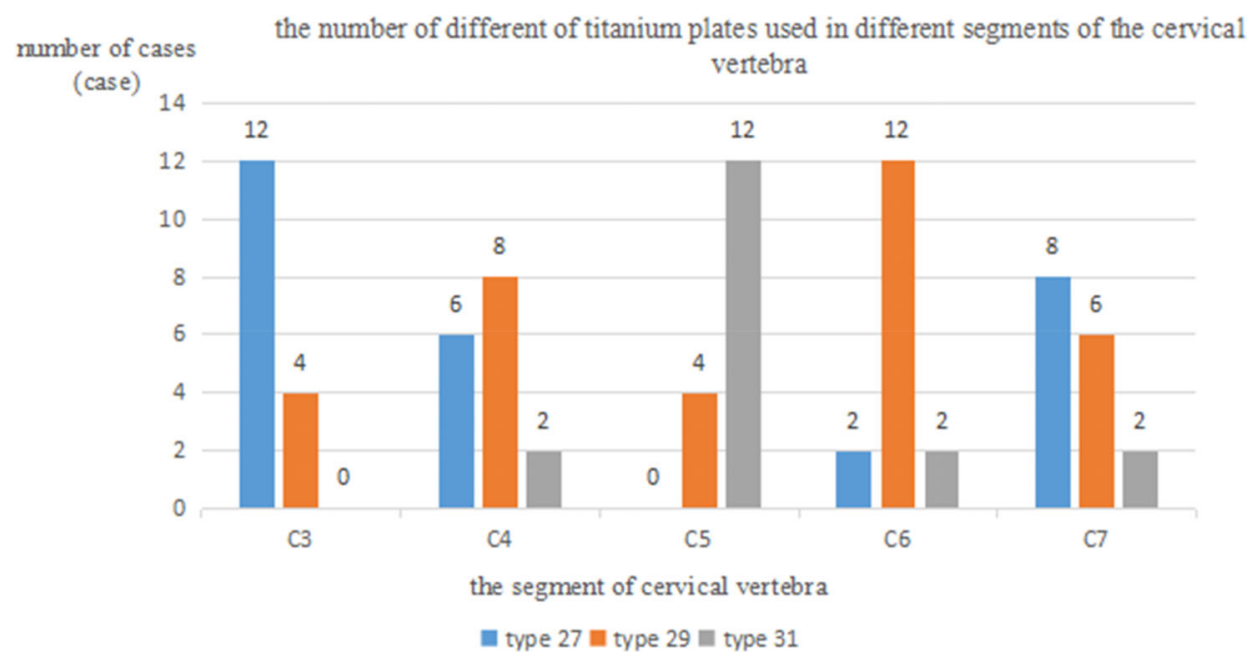

between the front end of the spinal cord and the posterior wall of the vertebral body was recorded as "C." Thus, the distance of the cervical spinal cord was defined as C-c.

\section{Statistical Methods}

All data were statistically analyzed by SPSS version 17.0 software. Data are expressed as the mean plus/minus the standard deviation about the mean $(\hat{X} \pm S D)$. Pearson's correlation analysis was performed to observe the relationship between the different segmental lamina posterior distances, spinal canal enlargement, and backward drift distance of the cervical spinal cord with different specifications of microtitanium plates. Pearson's correlation analysis was also used to peform a linear regression to establish a regression equation of the measured data.

\section{Results}

A 1116 patients were safely and successfully treated by surgery. The clinical outcome was good following the surgical procedure. One of the patients had symptoms of C5 nerve root palsy, and the painful symptoms were significantly relieved by conservative treatments, such as nutritional nerve treatment. The symptoms disappeared completely after 2 months postsurgery (Fig. 7).

The following are the number of titanium plates used in the cervical vertebrae and the relationship between the different cervical vertebra segment-related spinal canal parameters and microtitanium plate specifications. The posterior laminar distance of each segment, the enlarged area of the spinal canal, and the posterior movement distance of the cervical spinal cord increased with increasing size of the microtitanium plate, as shown in Table 1 and fig. 8 .

Pearson's correlation analysis was used to compare the relationship between the lamina posterior distance, spinal canal enlargement, cervical spinal cord backward drift distance and titanium plates with different specifications (Table 2). It was found that the different specifications of microtitanium plates had the following significant effects on the posterior movement distance of the lamina: $\mathrm{C}_{3}$ was 0.771 $(P<0.05), \mathrm{C}_{4}$ was $0.863(P<0.05), \mathrm{C}_{5}$ was $0.823(P<0.05)$, $\mathrm{C}_{6}$ was $0.842(P<0.05)$, and $\mathrm{C}_{7}$ was $0.869(P<0.05)$. The enlarged area of the $\mathrm{C}_{3}$ spinal canal was $0.789(P<0.05), \mathrm{C}_{4}$ was $0.862(P<0.05), \mathrm{C}_{5}$ was $0.845(P<0.05), \mathrm{C}_{6}$ was 0.880 $(P<0.05)$, and $\mathrm{C}_{7}$ was $0.784(P<0.05)$. The backward drift distance of the cervical spinal cord at $\mathrm{C}_{3}$ was $0.829(P$ $<0.05), \mathrm{C}_{4}$ was $0.908(P<0.05), \mathrm{C}_{5}$ was $0.896(P<0.05), \mathrm{C}_{6}$ was $0.935(P<0.05)$ and $\mathrm{C}_{7}$ was $0.852(P<0.05)$.

Establishing correlation equations. The regression equations for the different posterior distances of the cervical vertebrae and the microtitanium plate specifications are as follows:

$\mathrm{C}_{3}: \hat{Y}=0.067+2.326 \mathrm{X}\left(\mathrm{R}^{2}=0.594\right), \mathrm{C}_{4}: \hat{Y}=0.086$ $+4.335 \mathrm{X}\left(\mathrm{R}^{2}=0.746\right), \mathrm{C}_{5}: \hat{Y}=0.337+1.698 \mathrm{X}\left(\mathrm{R}^{2}=\right.$ $0.678), \mathrm{C}_{6}: \hat{Y}=0.219+3.592 \mathrm{X}\left(\mathrm{R}^{2}=0.710\right), \mathrm{C}_{7}: \hat{Y}=0.146$ $+5.628 \mathrm{X}\left(\mathrm{R}^{2}=0.755\right)$.

The regression equations for the different cervical segmental canal enlargement areas and microtitanium plate specifications are as follows:

$\mathrm{C}_{3}: \hat{Y}=2.065+35.455 \mathrm{X}\left(\mathrm{R}^{2}=0.622\right), \mathrm{C}_{4}: \hat{Y}=3.420$ $+62.750 \mathrm{X}\left(\mathrm{R}^{2}=0.743\right), \mathrm{C}_{5}: \hat{Y}=6.912+30.373 \mathrm{X}\left(\mathrm{R}^{2}\right.$ $=0.713), \mathrm{C} 6: \hat{Y}=3.955+77.175 \mathrm{X}\left(\mathrm{R}^{2}=0.774\right), \mathrm{C} 7: \hat{Y}=$ $5.405+0.952 \mathrm{X}\left(\mathrm{R}^{2}=0.614\right)$.

The regression equations for the backward drift distance of the cervical spine segments and the specifications of the microtitanium plates are shown below:

$\mathrm{C}_{3}: \hat{Y}=0.039+2.230 \mathrm{X}\left(\mathrm{R}^{2}=0.686\right), \mathrm{C}_{4}: \hat{Y}=0.084$ $+1.265 \mathrm{X}\left(\mathrm{R}^{2}=0.824\right), \mathrm{C}_{5}: \hat{Y}=0.098+1.685 \mathrm{X}\left(\mathrm{R}^{2}\right.$ $=0.802), \mathrm{C}_{6}: \hat{Y}=0.068+2.189 \mathrm{X}\left(\mathrm{R}^{2}=0.874\right), \mathrm{C}_{7}: \hat{Y}=$ $0.086+1.545 \mathrm{X}\left(\mathrm{R}^{2}=0.727\right)$.

\section{Discussion}

\section{Effectiveness of the Cervical Vertebral Dome Expansion Laminoplasty}

The cervical vertebral dome expansion laminoplasty was performed by moving the lamina as a whole and using a custom 
ORTHOPAEDIC SURGERY

Volume $13 \cdot$ Number 7 • OCTOBER, 2021
Cervical Vertebral Dome Expansion Laminoplasty

\begin{abstract}
microtitanium plate in the $\mathrm{C}_{6}$ segment, or $27 \times 4.5 \times 1$; four holes the microtitanium plate in the $\mathrm{C}_{7}$ segment.

Similarly, according to the regression equations obtained in this study, the operator can calculate and predict the parameters of the posterior movement distance of the cervical vertebrae obtained when using different sizes of microtitanium plates for different segments of the cervical vertebra; thus, it is possible to select the most suitable microtitanium plate for surgical treatment.

In this study, one patient developed $\mathrm{C}_{5}$ nerve root palsy after surgery. We consider that it was related to the excessive distance of the posterior lamina movement caused by the selection of a microtitanium plate that was too large during the operation, thus causing an excessive drift of the cervical spinal cord. Imagama et al. ${ }^{11}$ found that 43 patients had obvious symptoms of $\mathrm{C}_{5}$ nerve root palsy through an imaging study of 1858 patients undergoing posterior cervical surgery. The average distance of the cervical spinal cord was $3.9 \mathrm{~mm}$. In this patient, a miniature titanium plate with a specification of $31 \times 4.5 \times 1$; four holes were selected in the $\mathrm{C}_{5}$ segment. The posterior vertebral plate distance was $12.79 \mathrm{~mm}$, the enlarged area of the spinal canal was $184.56 \mathrm{~mm}^{2}$, and the cervical spinal cord was moved backward by $5.13 \mathrm{~mm}$. Through the regression equation, we found that for this patient in this cervical segment, the selection of a microtitanium plate with a specification of $29 \times 4.5 \times 1$; four holes could achieve the ideal clinical effect, which indicates the necessity of this study.

This study further clarified the most suitable titanium plate size for use in different cervical segments. Based on the results of the study, we recommend the use of the cervical vertebral dome expansion laminoplasty procedure for the treatment of cervical spondylosis with the use of the following microtitanium plates: $27 \times 4.5 \times 1$; four holes in the $\mathrm{C}_{3}$ segment, $29 \times 4.5 \times 1$; four holes in the $\mathrm{C}_{4}$ segment, $31 \times 4.5$ $\times 1$; four holes in the $\mathrm{C}_{5}$ segment, $29 \times 4.5 \times 1$; four holes in the $\mathrm{C}_{6}$ segment, and $27 \times 4.5 \times 1$; four holes in the $\mathrm{C}_{7}$ segment. Of course, when the impact of the surgical operation is excluded $^{12}$, the specifications of the microtitanium plate should be flexibly selected according to the individual anatomy of the patient and the pathological condition. In summary, the cervical vertebral dome expansion laminoplasty can effectively increase the posterior lamina distance, enlarged area of the spinal canal, and backward drift distance of the spinal cord. In addition, the custom dome-type microtitanium plates plays a vital role in the strong internal fixation of the cervical vertebra.

\section{Limitations}

The sample size in this study was relatively small. Therefore, future studies should compare the data of more patients. The regression equation between the changes in the imaging data of the cervical spinal canal and the specifications of the microtitanium plate was obtained. However, some patients with congenital cervical spinal stenosis cannot be generalized. Preoperative evaluation should be carried out based on actual imaging data to prevent related complications.

the $\mathrm{C}_{4}$ segment, the $31 \times 4.5 \times 1$; four holes microtitanium plate in the $\mathrm{C}_{5}$ segment, the $29 \times 4.5 \times 1 ; 4$ holes achieve a posterior lamina movement of $9.830 \pm 0.251 \mathrm{~mm}$ $\pm 0.071 \mathrm{~mm}$. The $\mathrm{C}_{7}$ segment uses a microtitanium plate specification of $31 \times 4.5 \times 1$; four holes, which can achieve a posterior lamina movement of $9.582 \pm 0.100 \mathrm{~mm}$ and a

The use of microtitanium plates with a specification of $27 \times 4.5 \times 1$; four holes in the $\mathrm{C}_{3}$ segment can achieve good clinical results. The same clinical effect can be achieved by using the $29 \times 4.5 \times 1$; four holes microtitanium plate in

fixation. At the same time, the volume of the spinal canal alleviating clinical symptoms; thus, the clinical curative effect $\pm 0.210 \mathrm{~mm}$ and a backward spinal cord movement distance of $4.737 \pm 0.044 \mathrm{~mm}$. The $\mathrm{C}_{6}$ segment uses a microtitanium plate specification of $31 \times 4.5 \times 1$; four holes, which can backward spinal movement distance of $4.160 \pm 0.071 \mathrm{~mm}$.
\end{abstract}




\section{References}

1. Traynelis VC, Arnold PM, Fourney DR, Bransford RJ, Fischer DJ, Skelly AC. Alternative procedures for the treatment of cervical spondylotic myelopathy: arthroplasty, oblique corpectomy, skip laminectomy: evaluation of comparative effectiveness and safety. Spine (Phila Pa 1976), 2013, 38: 210-231.

2. Nouri $A$, Tetreault $L$, Zamorano J, et al. Role of magnetic resonance imaging in predicting surgical outcome in patients with cervical spondylotic myelopathy.

Spine(Phila Pa 1976), 2015, 40: 171-178.

3. Hirabayashi K, Watanabe K, Wakano K, et al. Expansive open-door laminoplasty for cenical spinal stenotic myelopathy. Spine(Phila Pa 1976), 1983, 8: 693-699.

4. Sakaura $\mathrm{H}$, Hosono N, Mukai $\mathrm{Y}$, et al. C3-6 laminoplasty for cenical spondylotic myelopathy maintains satisfactory long-term surgical outcomes. Global Spine J,

2014, 4: 169-173.

5. Bin Z, Yibo Z, Lu X, et al. Research and application of cervical vertebral dome expansion laminoplasty. Chin J Orthop, 2019, 39: 604-612.

6. Lubelski D, Derkhshan A, Nowacki AS, et al. Predicting C5 palsy via the use of preoperative anatomic measurements. Spine J, 2014, 14: 1895-1901.
7. Sodeyama T, Goto S, Mochizuki M, et al. Effect of decompression enlargement laminoplasty for posterior shifting of the spinal cord. Spine(Phila $\mathrm{Pa}$ 1976), 1999, 24: 1527-1531.

8. Itoh T, Tsuji H. Technical improvements and results of laminoplasty for compressive myelopathy in the cervical spine. Spine(Phila Pa 1976), 1985, 10: 729-736.

9. Kohno K, Kumon Y, Oka Y, et al. Evaluation of prognostic factors following expansive laminoplasty for cervical spinal stenotic myelopathy. Surg Neurol, 1997, 48: 237-245.

10. Epstein NE. Surgical management of lumbar stenosis: decompression and indications for fusion. Neurosurgical Focus, 1997, 3: e1-e4.

11. Imagama $S$, Matsuyama $Y$, Yukawa $Y$, et al. $C 5$ palsy after cervical laminoplasty: a multicenter study. J Bone Joint Surg Br, 2010, 92: 393-400. 12. Hashimoto $M$, Mochizuki $M$, Aiba $A$, et al. $C 5$ palsy following anterior decompression and spinal fusion for cervical degenerative diseases. Eur Spine J, 2010, 19: 1702-1710. 